J. Opt. Fiber. Commun. Rep. 1,305-311 (2004) (C) 2004 Springer Science + Business Media Inc. DOI: 10.1007/s10297-004-0009-x

Editors: Curtis R. Menyuk, Andrea Galtarossa

Optical and Fiber
JOuna of
Communications Reports

\title{
Interaction of nonlinearity and polarization mode dispersion
}

\author{
Curtis R. Menyuk \\ University of Maryland Baltimore County \\ Computer Science and Electrical Engineering Department \\ 1000 Hilltop Circle, Baltimore, MD, 21250 USA \\ and \\ PhotonEx Corporation \\ 200 MetroWest Technology Park, Maynard, MA 01754 USA
}

\begin{abstract}
The derivation of the coupled nonlinear Schrödinger equation and the Manakov-PMD equation is reviewed. It is shown that the usual scalar nonlinear Schrödinger equation can be derived from the Manakov-PMD equation when polarization mode dispersion is negligible and the signal is initially in a single polarization state as a function of time. Applications of the Manakov-PMD equation to studies of the interaction of the Kerr nonlinearity with polarization mode dispersion are then discussed.
\end{abstract}

There has been a flood of recent work on polarization effects in optical fibers, most of it focused on polarization mode dispersion (PMD). There are good, practical reasons for this recent interest. As the data rate per channel increases, PMD becomes an increas-ingly important limitation in communication systems. However, my own interest in polarization issues was, at least originally, focused on more fundamental questions. Since nearly all the contributions in this volume focus on the more immediate practical issues that are a consequence of polarization effects, it seemed useful to me to focus this contribution on more fundamental issues. The nice thing about fundamental issues is that even though they attract less attention than immediate practical issues, they typically stay relevant longer and can impact practical issues $10-20$ years in the future.

Throughout the 1970s, a considerable body of work established the basic polarization properties of optical fibers. Much of this work was summarized by Kaminow in 1981 [1]. It was found that the birefringence $\Delta n / n$ is in the range $10^{-4}-10^{-9}$, with communication fibers in the range $10^{-6}-10^{-7}$. It was found that the intrinsic 
birefringence is almost entirely linear, even when the fiber is twisted, because of the very small value of the electro-optic tensor in glass [2]. Remarkably, there has been no change in either the range of the birefringence values or the helicity in optical fibers in the last 20 years, although many other fiber parameters have changed considerably. The fiber-induced PMD can now be made much smaller by spinning fibers as they are drawn; the effective areas of fibers can be made much larger or smaller, affecting the strength of the nonlinearity; the dispersion can be tailored; and losses can be made smaller.

In 1980, Mollenauer et al. [3] demonstrated the propagation of solitons in optical fibers for the first time. This work began a series of studies, continuing into the present day, that explored the interaction between chromatic dispersion and nonlinearity in optical fibers and their implications for communication systems. The theoretical basis for this work was the nonlinear Schrödinger equation, which had been derived by Hasegawa and Tappert in 1973 [4]. This work took no account of the birefringence in optical fibers; however, work by Botineau and Stolen [5] had made it clear that the interaction of birefringence and nonlinearity could be quite important. In their work, they measured the nonlinear polarization rotation and demonstrated its potential for nonlinear switching. As part of this work, they demonstrated that the ratio of the crossphase modulation and the self-phase modulation in optical fibers is $2 / 3$. I note that this coefficient is only expected to be $2 / 3$ if the fiber's birefringence is intrinsically linear. More generally, it equals $\left(2+2\left|\hat{\mathbf{e}}_{1} \cdot \hat{\mathbf{e}}_{2}\right|\right) /\left(2+\left|\hat{\mathbf{e}}_{1} \cdot \hat{\mathbf{e}}_{1}\right|\right)$, where $\hat{\mathbf{e}}_{1}$ and $\hat{\mathbf{e}}_{2}$ are the unit vectors for the birefringent eigenmodes [6]. Its value ranges from $2 / 3$ for linearly birefringent fibers to 2 for circularly birefringent fibers. Thus, the nonlinear light evolution yields information about the linear properties of the fibers-a point to which I shall return.

Given the experimental evidence of the importance of birefringence, it was natural to derive an equation that takes it into account. This equation, referred to as the coupled nonlinear Schrödinger equation, was first presented in 1987 [7]. In a form that is particularly useful for studying randomly varying birefringence, it may be written as

$$
\begin{aligned}
& i \frac{\partial \mathbf{u}}{\partial z}-i g \mathbf{u}+\left(\sigma_{3} \cos \theta+\sigma_{1} \sin \theta\right)\left(\Delta \beta \mathbf{u}+i \Delta \beta^{\prime} \frac{\partial \mathbf{u}}{\partial t}\right)-\frac{1}{2} \beta^{\prime \prime} \frac{\partial^{2} \mathbf{u}}{\partial t^{2}} \\
& \quad+\gamma\left[|\mathbf{u}|^{2} \mathbf{u}-\frac{1}{3}\left(\mathbf{u}^{\dagger} \sigma_{2} \mathbf{u}\right) \sigma_{2} \mathbf{u}\right]+0,
\end{aligned}
$$

where $z$ and $t$ are distance along the fiber and retarded time, respectively, while $\mathbf{u}(z, t)$ is the two-dimensional Stokes vector wave envelope. The parameter $g$ is the gain and loss in the fiber, $\theta / 2$ is the orientation angle of the axis of birefringence, $\Delta \beta$ is the birefringence, $\Delta \beta^{\prime}$ is the frequency derivative of the birefringence, $\beta^{\prime \prime}$ is the dispersion, and $\gamma$ is the Kerr coefficient. All parameters are evaluated at the carrier frequency. The $\sigma_{j}$ are the standard Pauli matrices. My original motivation for deriving this equation was that for deep theoretical reasons I had hypothesized that solitons would be robust in the presence of Hamiltonian perturbations [8]-[10]. It is beyond the scope of this contribution to discuss the origin of this hypothesis and what Hamiltonian perturbations are, except to note that when the birefringence is small, it is a Hamiltonian perturbation. What does it mean for the birefringence to be small? It means that the birefringent beat length should be large compared to the nonlinear scale length and the scale length for chromatic dispersion. Under these circumstances, theory [11] and experiment [12] show that solitons are robust in the presence of birefringence, as predicted by the 
robustness hypothesis. However, this limit is not the usual limit that applies in optical fiber communication systems. In these systems, the beat length is short compared to the scale lengths for nonlinearity and chromatic dispersion, as shown in Fig. 1. Under these circumstances, the coupled nonlinear Schrödinger equation indicates that solitons should be ripped apart-an effect that is not observed. Clearly, there is a mystery here.

In its broad outlines, the answer to this mystery, shortly after it first became apparent in 1988, was not long in coming. The birefringence in optical fibers is randomly varying. As long as the PMD scale length is long compared to the nonlinear and dispersive scale lengths, solitons will be robust [13]-[15]. The PMD scale length is the length over which PMD will differentially change the polarization states in the bandwidth of a signal so that the polarization state is no longer constant over the signal spectrum. This scale length is the same as the scale length over which PMD will cause a linear pulse to spread significantly [16]-[18].

However, a key question remained: Why and when should the nonlinear Schrödinger equation hold? Since the early, very brief and intuitive derivation of Hasegawa and Tappert [4], numerous derivations of the nonlinear Schrödinger equation have been published. Many of these derivations contain mathematical errors. Two of the most common are neglecting the $\partial^{2} / \partial z^{2}$ term that appears in Maxwell's equations, rather than eliminating it self-consistently, and dropping the $\nabla(\nabla \cdot \mathbf{E})$ term. However, Kodama [19] has published a derivation that takes into account these issues as well as numerous other issues and is, I believe, completely correct mathematically. However, this derivation shares with all others up to my recently published derivation [18] what I consider to be a conceptual flaw. All derivations up to [18] assumed that the core of an optical fiber is perfectly round and without stress and thus neglect the birefringence entirely. In fact, a physically correct derivation that is applicable to communication systems should take into account the large, but rapidly and randomly varying, birefringence. From a conceptual standpoint, the logical chain that one must use is as follows: Starting from Maxwell's equations, one must average over the rapid oscillations in the carrier wave to obtain the coupled nonlinear Schrödinger equation, which appears in the middle group of scale lengths that I show in Fig. 1. From there, one must average over the rapidly and randomly varying birefringence to obtain the Manakov-PMD equation that applies to the largest group of length scales in Fig. 1. When PMD can be neglected, and, in addition, the initial field is in a single polarization state as a function of time, then the nonlinear Schrödinger equation holds [18].

When my colleagues and I began to tackle this question in the early 1990s, the first issue that we faced was that we did not know how to model the randomly varying birefringence in the fibers. In his analytical work, Poole [20] used a model that assumed weak off-diagonal coupling between the axes of birefringence. This model was first used to describe polarization-maintaining fiber, for which it is physically reasonable [21], but it was less obvious to my colleagues and I that it is physically reasonable for communication fibers in which we knew that the orientation of the birefringence axes had to fairly rapidly cover all possible values uniformly. How did we know that? If the opposite held, then, as noted previously, our early work had make it clear that the birefringence would rip solitons apart—something that is not observed. For numerical work, Poole et al. [22] introduced a rotating waveplate model. It is closely related to the coarse step method, which was introduced in [14] and [15] to allow users to deal effectively with systems in which nonlinearity, chromatic dispersion, and randomly varying birefringence are all present. A difficulty in principle with the rotating wave- 


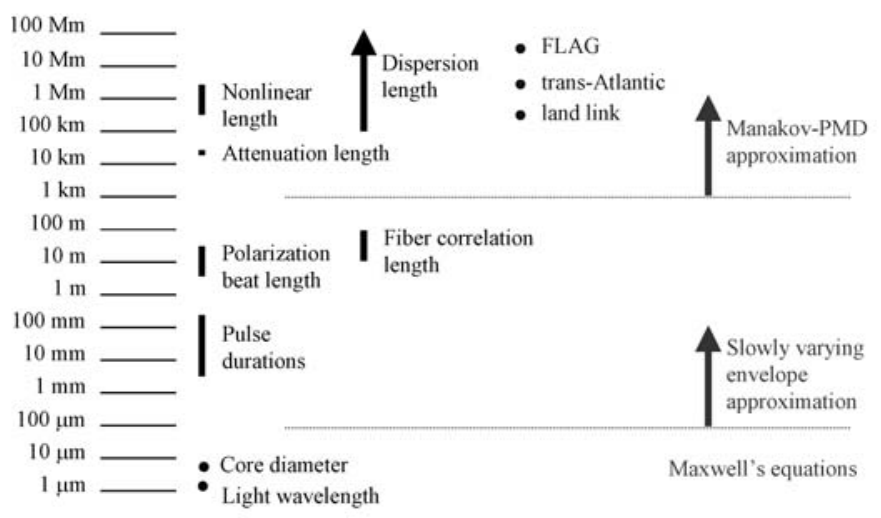

Fig. 1. Illustration of the key length scales in optical fiber transmission systems.

plate model that the models introduced in [14] and [15] avoid is that if the beat length is resonant with birefringent fiber lengths, then a linear input polarization state can only transform to other linearly polarized states. Thus, the randomly rotating waveplate model is not suitable for modeling broadband wavelength division multiplexed systems. By contrast, the coarse step models are widely used in both commercial and non-commercial, full-system simulators.

A key issue in deriving the Manakov-PMD equation is determining the statistics of the evolution of polarization states on the Poincaré sphere. Since the statistical variation of the birefringence in optical fibers was unknown, Wai and Menyuk [23] proposed two physical models. In the first model, the birefringence is fixed and its orientation is allowed to vary. In the second, the change in the index of refraction due to the birefringence is assumed to be Gaussian-distributed in both transverse directions of the fiber. Our original thought was that the two models might make different predictions for the field evolution that would allow one to determine experimentally which underlying fiber model is correct. In fact, the two models make nearly identical predictions. Thus, the issue remained unresolved until the recent polarization optical time domain reflectrome-try measurements by Galtarossa et al. [24] showed, in combination with earlier results indicating that the fiber birefringence is linear, that the second model is correct. Alex Wai and I found that the evolution on the Poincare sphere is anisotropic and that it depends on two parameters, the average fiber beat length $L_{b}$ and the fiber decorrelation length $L_{f}$. The field evolution on the Poincare sphere is characterized by three parameters, an azimuthal diffusion length $L_{\mathrm{az}}$, an equatorial diffusion length in a frame that is fixed $L_{\text {eq,fixed, and an equatorial diffusion length in a frame that rotates }}$ with the axes of birefringence, $L_{\text {eq,rot }}$. Both Laz and Leq,fixed are proportional to $L_{f}$ when $L_{b}$ ? $L_{f}$ and to $L_{b}^{2} / L_{f}$ when $L_{f}$ ? $L_{b}$. By contrast, $L_{\text {eq, rot }}$ is proportional to $L_{f}$, regardless of $L_{b}$.

To average over the coupled nonlinear Schrödinger equation to obtain the ManakovPMD equation, one proceeds in two steps [18], [23]. In the first step, one makes the transformation

$$
\tilde{\mathbf{v}}(z, \omega)=\left\{\mathbf{I} \cos [\theta(z) / 2]+\sigma_{2} \sin [\theta(z) / 2]\right\} \tilde{\mathbf{u}}(z, \omega),
$$


where the tilde indicates the Fourier transform of the time domain quantities, and $\mathbf{I}$ is the $2 \times 2$ identity matrix. This transformation would diagonalize the linear portion of the coupled nonlinear Schrödinger equation in the absence of a $z$-varying $\theta$. I note that $\omega$ is defined with respect to the carrier frequency. In the second step, we freeze the $\omega=0$ motion by making the transformation $\tilde{\mathbf{v}}(z, \omega)=\mathbf{T}^{-1} \tilde{\mathbf{u}}(z, \omega)=$, where

$$
\left.i \frac{\partial \mathbf{T}(z)}{\partial z}+\left\{\Delta \beta \sigma_{3}+\left[\theta_{z}(z) / 2\right] \sigma_{2}\right\} \mathbf{T}(z)=\right) ; \quad \mathbf{T}(z=0)=\mathbf{I},
$$

and $\theta_{z}$ indicates the $z$-derivative of $\theta$. Returning to the time domain, we obtain the Manakov-PMD equation,

$$
\begin{aligned}
i \frac{\partial \mathbf{w}}{\partial z} & -i g \mathbf{w}-\frac{1}{2} \beta^{\prime \prime} \frac{\partial^{2} \mathbf{w}}{\partial t^{2}}+\frac{8}{9} \gamma|\mathbf{w}|^{2} \mathbf{w} \\
& =-i \Delta \beta^{\prime} \bar{\sigma}_{3} \frac{\partial \mathbf{w}}{\partial t}+\frac{1}{3} \gamma\left[\left(\mathbf{w}^{\dagger} \bar{\sigma}_{2} \mathbf{w}\right) \bar{\sigma}_{2} \mathbf{w}-\frac{1}{3}|\mathbf{w}|^{2} \mathbf{w}\right]
\end{aligned}
$$

where $\bar{\sigma}_{j}=\mathbf{T}^{-1} \sigma_{j} \mathbf{T}$. On the left-hand side of Eq. (4), we have the slowly varying contribution. On the right-hand side, we have the rapidly varying residual contributions. The first residual term is linear and leads to the usual linear PMD. The length scale on which averaging occurs equals $L_{\text {eq,rot }} \sim L_{f}$, regardless of $L_{b}$. However, the accumulated differential group delay (DGD) is proportional to the birefringence; so, the expected value of the DGD is proportional to $L_{f}^{1 / 2} / L_{b}$ over lengths that are large compared to $L_{f}$. The second residual term is nonlinear and leads to an effect that my colleagues and I refer to as nonlinear PMD. Marcuse et al. [25] showed that this term is negligible in communication systems. However, it can be important in short-pulse systems, and Arend et al. [26] observed its effect in fiber loop mirrors. Interestingly, the strength of this effect is proportional to $L_{\mathrm{az}}$, in contrast to the usual linear PMD, whose strength is proportional to $L_{\text {eq,rot }}$.

While the nonlinear PMD can be neglected in present-day communication systems, the same is not true for the interaction of nonlinearity and the usual linear PMD. One example of this effect is that a nonlinearly induced pulse chirp can interact with higher-order PMD to lead to additional pulse spreading beyond the spreading that is induced by first-order PMD or can induce pulse compression. This effect was predicted by Ibragimov et al. [27] and was recently observed by Möller et al. [28]. A second example of this effect is that nonlinear polarization rotation induced by wavelength division multiplexed interactions can lead to fast variations of the polarization states that impede the ability of standard PMD compensators to mitigate signal degradation that is due to PMD. This effect was observed by Khosravani et al. [29] and by Lee et al. [30]. It is my view that much work remains to be done to explore the interaction of nonlinearity and PMD and that it is a very interesting area for future research.

In conclusion, much of my own work on polarization effects in optical fibers was motivated by a desire to understand what the basic equations are that govern light propagation in optical fibers on the length and time scales that are important for communication systems. This work led to the derivation of the coupled nonlinear Schrödinger equation that governs the evolution on the intermediate length scale of Fig. 1 and to the Manakov-PMD equation that governs the evolution on the long length scale of Fig. 1. From this work, we find that the usual scalar nonlinear Schrödinger equation and its variants will hold when PMD is negligible and the signal is launched 
in a single polarization state. Establishing the Manakov-PMD equation was important step forward. It includes all the physical phenomena of importance in optical fiber communications except the Brillouin and Raman nonlinearities, device effects like polarization dependent loss, and amplified spontaneous emission noise from the amplifiers. The Manakov-PMD equation can easily be modified to include these effects. It contains a rich set of phenomena, many of practical importance, which we have only begun to explore.

\section{References}

[1] I. P. Kaminow, “Polarization in optical fibers,” IEEE J. Quantum Electron., 17, 15-22 (1981).

[2] S. C. Rashleigh, "Origins and control of polarization effects in single-mode fibers," J. Lightwave Technol., 1, 312-331 (1983).

[3] L. F. Mollenauer, R. H. Stolen, and J. P. Gordon, "Experimental observation of picosecond pulse narrowing and solitons in optical fibers," Phys. Rev. Lett., 45, 1095-1098 (1980).

[4] A. Hasegawa and F. Tappert, "Transmission of stationary nonlinear optical pulses in dispersive dielectric fibers. I. Anomalous dispersion,” Appl. Phys. Lett., 23, 142-144 (1973).

[5] J. Botineau and R. H. Stolen, "Effect of polarization on spectral broadening in optical fibers," J. Opt. Soc. Am., 72, 1592-1596 (1982). See also, R. H. Stolen, J. Botineau, and A. Ashkin, "Intensity discrimination of optical pulses with birefringent fibers," Opt. Lett., 7, 512-514 (1982).

[6] C. R. Menyuk, "Pulse propagation in an elliptically birefringent Kerr medium," IEEE J. Quantum Electron., 25, 2674-2682 (1989).

[7] C. R. Menyuk, "Nonlinear pulse propagation in birefringent optical fibers," IEEE J. Quantum Electron., 23, 174-176 (1987).

[8] C. R. Menyuk, "Origin of solitons in the 'real' world," Phys. Rev. A, 33, 4367-4374 (1986).

[9] C. R. Menyuk, "Why are solitons robust in optical fibers," Guided Wave Nonlinear Optics, D. Ostrowsky and R. Reinisch, eds. (Kluwer, Dordrecht, Netherlands, 1992), pp.457-499.

[10] C. R. Menyuk, "Soliton robustness in optical fibers," J. Opt. Soc. Am. B, 10, 1585-1591 (1993).

[11] C. R. Menyuk, "Stability of solitons in birefringent optical fibers. I: Equal propagation amplitudes," Opt. Lett., 12, 614-616 (1987); "Stability of solitons in birefringent optical fibers. II. Arbitrary amplitudes," J. Opt. Soc. Am. B, 5, 392-402 (1988).

[12] M. N. Islam, C. D. Poole, and J. P. Gordon, "Soliton trapping in birefringent optical fibers," Opt. Lett., 14, 1011-1013 (1989).

[13] L. F. Mollenauer, K. Smith, J. P. Gordon, and C. R. Menyuk, "Resistance of solitons to the effects of polarization dispersion in optical fibers," Opt. Lett., 14, 1219-1221 (1989).

[14] P. K. A. Wai, C. R. Menyuk, and H. H. Chen, "Stability of Solitons in Randomly Varying Birefringent Fibers," Opt. Lett., 16, 1231-1233 (1991). 
[15] S. G. Evangelides, L. F. Mollenauer, and J. P. Gordon, "Polarization multiplexing with solitons," J. Lightwave Technol., 10, 28-34, 1992.

[16] C. D. Poole and R. E. Wagner, "Phenomenological approach to polarisation dispersion in long single-mode fibres," Electron. Lett., 22, 1029-1030 (1986).

[17] M. Karlsson, "Polarization mode dispersion-induced pulse broadening in optical fibers," Opt. Lett., 23, 688-690 (1998).

[18] C. R. Menyuk, "Application of multiple length-scale methods to the study of optical fiber transmission," J. Engin. Math., 36, 113-136 (1999).

[19] Y. Kodama, "Optical solitons in a monomode fiber," J. Stat. Phys., 39, 597-614 (1985). See, also, A. Hasegawa and Y. Kodama, Solitons in Optical Communications (Clarendon, Oxford, UK, 1995), Chap. 1.

[20] C. D. Poole, "Statistical treatment of polarization dispersion in single-mode fiber," Opt. Lett., 13, 687-689 (1988).

[21] S. C. Rashleigh, W. K. Burns, R. P. Moeller, and R. Ulrich, "Polarization holding in birefringent single-mode fibers," Opt. Lett., 7, 40-42 (1982).

[22] C. D. Poole, J. H. Winters, and J. A. Nagel, "Dynamical equation for polarization dispersion," Opt. Lett., 16, 372-374 (1991).

[23] P. K. A. Wai and C. R. Menyuk, "Polarization mode dispersion, decorrelation, and diffusion in optical fibers with randomly varying birefringence," J. Lightwave Technol., 14, 148-157 (1996).

[24] F. Corsi, A. Galtarossa, and L. Palmieri, "Beat-length characterization based on backscattering analysis in randomly perturbed single-mode fibers," J. Lightwave Technol., 17, 11721183 (1999); A. Galtarossa, L. Palmieri, M. Schiano, and T. Tambosso, "Statistical characterization of fiber random birefringence," Opt. Lett., 25, 1322-1324 (2000).

[25] D. Marcuse, C. R. Menyuk, and P. K. A. Wai, "Application of the Manakov-PMD equation to studies of signal propagation in optical fibers with randomly varying birefringence," J. Lightwave Technol., 15, 1735-1746 (1997).

[26] M. F. Arend, M. L. Dennis, I. N. Duling, E. A. Golovchenko, A. N. Pilipetskii, and C. R. Menyuk, "Nonlinear-optical loop mirror demultiplexer using a random birefringence fiber: Comparisons between theory and experiments," Opt. Lett., 22, 886-888 (1997).

[27] E. Ibragimov, C. R. Menyuk, and W. Kath, "PMD-induced reduction of nonlinear penalties in terrestrial optical fiber transmission," Optical Fiber Communication Conference 2000, Baltimore, MD, paper WL3.

[28] L. Möller, Y. Su, G. Raybon, S. Chandrasekhar, and L. L. Buhl, "Penalty interference of nonlinear intra-channel effects and PMD in ultra high-speed TDM systems," Electron. Lett., 38, 281-283 (2002). Note that Eqs. (1) and (2) contain small errors.

[29] R. Khosravani, Y. Xie, L. S. Yan, Y. W. Song, A. E. Willner, and C. R. Menyuk, "Limitations to First-Order PMD Compensation in WDM Systems Due to XPM-Induced PSP Changes," Optical Fiber Communications Conference 2001, Anaheim, CA, paper WAA5.

[30] J. H. Lee, K. J. Park, C. H. Kim, and Y. C. Chung, "Impact of nonlinear crosstalk on optical PMD compensation," Optical Fiber Communications Conference 2002, Anaheim, CA, paper ThI2. 